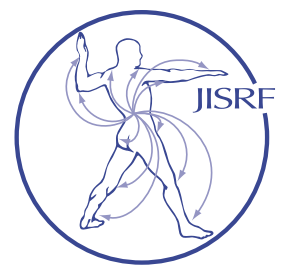

\title{
A Literature-Based Resource for the Development of Outpatient Arthroplasty Patient Selection Criteria
}

\author{
Gondusky, $\mathrm{J}^{1}$; Pahapill, $\mathrm{R}^{2}$; Coulson, $\mathrm{C}^{2}$
}

\begin{abstract}
Abstact
Total joint arthroplasty (TJA) is moving towards the outpatient setting. Teams must develop patient selection criteria to ensure appropriate candidates are treated at the optimal site of care. Protocols and recommendations have been developed to aid care teams in developing patient selection criteria, but these come from multiple disparate sources. We review the available literature on patient selection criteria and optimization in the outpatient TJA population, and synthesize this information into a workable format for care design. We hope to provide a resource to stakeholders that can be tailored to their unique outpatient facility.
\end{abstract}

\section{Background}

Total joint arthroplasty (TJA) is moving toward the outpatient setting. The change has been facilitated by improved perioperative protocols and anesthesia techniques, and increasingly embraced by patients, surgeons, and insurers. Adaptation of protocols from the hospital inpatient to the outpatient setting requires a focused effort on the part of the care team to develop pathways that ensure continued safety, quality, satisfaction, and cost containment. An aspect of this care design needs to be a thoughtful consideration of who to select for candidacy at an outpatient facility. We attempt to provide a review of the available literature on patient selection criteria for outpatient TJA, and we synthesize this data in attempts to provide a reference to care teams attempting to create selection criteria appropriate for their unique facility. In addition, given its applicability to patient selection, we present a review of modifiable risk factors that affect TJA outcome, as well as patient optimization opportunities.

\section{Materials \& Methods}

A review of published material related to outpatient, rapid discharge or same day TJA was queried, with specific focus on outpatient surgery, same day discharge, and patient optimization. We searched the PubMed database for articles published between January 1, 2015, and December 31, 2019, in order to focus on relevant, modern studies. Articles that included a specific focus on outpatient surgery, same day discharge, and patient optimization about the TJA episode were included. Articles focusing on upper extremity surgery, or without defined tangent variables allowing for comparison, such as generic opinion articles, were excluded (Fig. 1). The most relevant available articles were individually determined and reviewed, with a goal of enough focused studies analyzed to ensure adequate breadth of the topic. [1-19] Articles were grouped into those providing data on selection criteria, failed dis-

\footnotetext{
Keywords: Total joint arthroplasty, outpatient, same day discharge, selection criteria, patient optimization Level of Evidence: IV
} 
charge, or patient optimization. Studies focused on selection criteria were reviewed, with selection variables extracted. These were compared across studies. The relative paucity of studies and overall low levels of evidence did not allow for a true weighting of variables as is possible in more robust systematic reviews. Hence, the studies were synthesized grossly to be able to present an overall review of published literature on the topic. Inclusion and exclusion criteria for selection were grouped according to variable for attempted presentation in a consolidated format. Optimization and discharge failure data were synthesized and are presented descriptively.

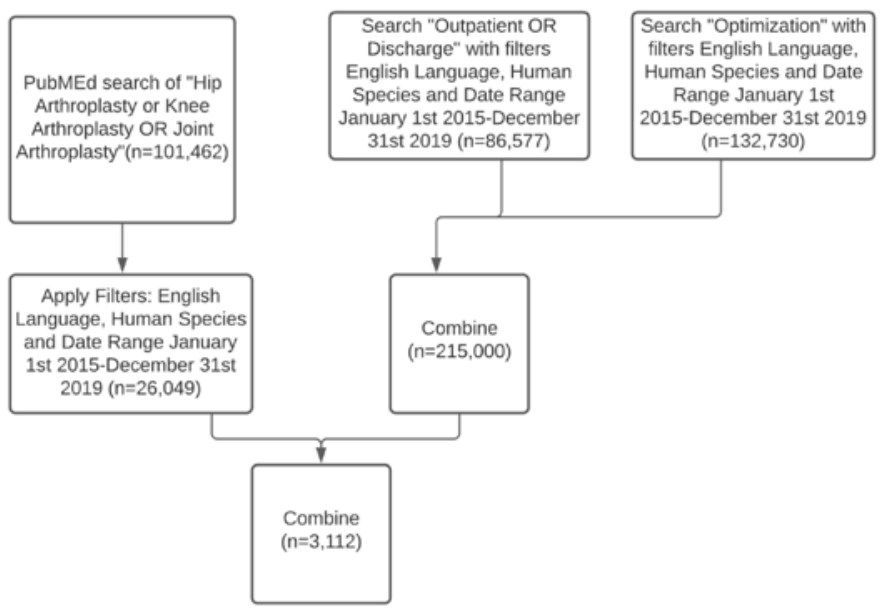

Figure 1: Search methodology for relevant articles

\section{Results}

Multiple authors have reported on their results with outpatient TJA. [1-19] Some studies select patient inclusion and exclusion criteria, and then determine the results of patient outcomes based on this selection. Others attempt to define appropriate criteria based on a retrospective review of successful day of surgery discharge after TJA. Scoring systems have also been promoted to aid the team in appropriate selection of the outpatient TJA candidate. $[\underline{7}, \underline{8}]$ Given the heterogeneity of the literature on the topic, we attempted to provide some synthesis to guide stakeholders.

Certain variables are consistently addressed in the literature. These are summarized in Table 1. Many authors note specific but less universal recommendations for exclusion. These are valuable for consideration and are presented in Table 2.
Table 1: A list of variables consistently noted by various authors.

\begin{tabular}{|l|l|}
\hline Patient Variable & Range of Exclusion \\
\hline Age (yrs.) & $>65-75[\underline{1}, \underline{\underline{2}}, \underline{4}, \underline{9}]$ \\
\hline ASA Class* & $\geq 3[\underline{1}, \underline{9}-\underline{11}]$ \\
\hline BMI $(\mathrm{kg} / \mathrm{m} 2)$ & $>35-40[\underline{1}-\underline{3}, \underline{9}, \underline{10}, \underline{12}]$ \\
\hline $\begin{array}{l}\text { Preoperative Hemoglobin }(\mathrm{g} / \mathrm{dL}), \\
\text { Hematocrit }\end{array}$ & $<10-12[\underline{1}-\underline{3}, \underline{9}, \underline{11}],<30 \%[\underline{4}]$ \\
\hline Glucose on Day of Surgery $(\mathrm{mg} / \mathrm{dL})$ & $>180-200[\underline{9}, \underline{12}, \underline{13}]$ \\
\hline Hemoglobin A1C $(\%)$ & $>7.5[\underline{11}, \underline{14}]$ \\
\hline Timed Up and Go Test (s) & $>10[\underline{5}]$ \\
\hline Creatinine (mg/dL) & $>2[\underline{14}]$ \\
\hline Sodium (mEq/L) & $<126[\underline{14}]$ \\
\hline Potassium (mmol/L) & $<3[\underline{14}]$ \\
\hline $\begin{array}{l}\text { STOP-BANG score for Obstructive } \\
\text { Sleep Apnea (OSA) }\end{array}$ & $>5[\underline{9}]$ \\
\hline
\end{tabular}

Table 1 reveals variable exclusion ranges noted by some authors. *ASA 3 was considered appropriate criteria for outpatient TJA by most authors. In a review of 3,444 charts, over $1 / 3$ of ASA 3 patients were otherwise deemed eligible for TJA in the ambulatory surgical center when their composite screening criteria were utilized. [10]

Table 2: A list of general exclusion criteria noted by some authors.

\begin{tabular}{|l|}
\hline Other General Exclusion Criteria to Consider \\
\hline Revision Surgery $[\underline{1}, \underline{2}]$ \\
\hline Bilateral Arthroplasty $[\underline{1}, \underline{2}]$ \\
\hline Inability to Ambulate Without a Walker $[\underline{1}, \underline{2}]$ \\
\hline $\begin{array}{l}\text { Significant Cardiopulmonary History }[\underline{1}-\underline{4}, \underline{9}-\underline{11}] \text { (see article text for } \\
\text { definition) }\end{array}$ \\
\hline History of Thromboembolic Event $[\underline{4}, \underline{9}-\underline{11}]$ \\
\hline Chronic Anticoagulation $[\underline{3}, \underline{9}]$ \\
\hline Presence of Obstructive Sleep Apnea $[\underline{3}, \underline{9}, \underline{10}, \underline{15}]$ \\
\hline Chronic Liver Disease (Childs class B or worse) $[\underline{[}]$ \\
\hline Cerebral Vascular Disease $[\underline{9}]$ \\
\hline Chronic pre-operative opioid use/addiction $[\underline{1}, \underline{2}]$ \\
\hline $\begin{array}{l}\text { Inadequate assistance at home after discharge1/lack of support } \\
{[\underline{2}, \underline{3}, \underline{15}]}\end{array}$ \\
\hline Cognitive Deficiencies $[\underline{15}]$ \\
\hline
\end{tabular}

Significant cardiopulmonary history is important to define, as many patients present with some history of diagnosis in these systems, and one needs tangible guidelines to aid selection criteria. This is defined in the reviewed studies as "no history of cardiopulmonary disease that would necessitate inpatient monitoring after surgery", [1] no CABG or stent placement within the last 6 months, [4] no history of coronary arterial disease or arrhythmias, []ㅡ 
no history of MI or CAD with PCI or CABG within the last one year, [10] and no oxygen or COPD/asthma steroid dependency. [9]

Reasons for failed day of surgery discharge can help modify selection criteria and focus efforts on care design. A review of available literature reveals some common issues. Fraser et al. noted the importance of preoperative education and planning, as most of their patients failed same day discharge due to patient preference, with patient concern about being "tired, safety concerns at home, or a long drive home". [1] They also found a significant correlation with failure and the number of patient-reported allergies, anxiety, depression, OSA and rheumatoid arthritis (RA). From a medical standpoint, they had a large percentage fail due to dizziness, vasovagal episodes, and/or hypotension. [1] DeCook noted discharge delays being predictable due to hyper or hypotension, over sedation, urinary retention, nausea or vomiting, pain and social support issues. [19] Richards et al. reported that out of 7 failures, 5 were due to hypotension, one due to pain, and one urinary retention. [5] Goyal et al. reported similar issues limiting day of surgery discharge with overall $24 \%$ failing. Older women had the most significant issue with prolonged length of stay in their study. [2]

A review of optimization strategies is also valuable to consider in determining care design and selection criteria. A few authors present excellent and comprehensive reviews of the topic. $[\underline{13}, \underline{14}, \underline{16}-\underline{18}]$ While a thorough review of patient optimization is beyond the scope of this article, we believe the basic variables addressed are worthy of note, in order to alert the care team for their consideration. These are presented in Table 3.

Yu et al, Boraiah et al and Kim et al describe a surgeon-led screening and optimization initiative. [16-18] They identify eight risk factor categories that are modifiable. These 8 categories include infection, smoking, obesity/malnutrition, cardiovascular disease, history of VTED, neurocognitive/psychiatric substance-related problems, physical deconditioning, and diabetes/malnutrition. [16] Using their protocol, they noted a lower readmission rate, cost and skilled nursing facility discharge (SNF DC). [16]

\section{Discussion}

Primary TJA should be "successful" and reproducible. "Success" should be defined as reflecting the "triple aim" initiative promoted by the IHI institute for HC improvement: better care for individuals (quality/satisfaction/patient experience), better health for populations, and lower per capita costs. As a huge annual healthcare expenditure, that has proven to be of value to society, $[\underline{21}, \underline{22}]$ progressing TJA to the outpatient setting should be seen as a way to fulfill this "triple aim."

Care design is critical to successful implementation of an outpatient arthroplasty program. Patient selection criteria represent an important aspect of the care design pathway. Since it is impossible to extricate patient optimization and risk factor modification from the selection decision-making process, it would seem to make sense to consider these aspects of patient care at the time of considering patient selection criteria. It would also be important to consider why patients fail in the outpatient setting in order to understand how proposed selection criteria or preoperative pathways may need to be altered in order to achieve outpatient arthroplasty success. It is also important to constantly consider one's particular facility. Patient mix, available resources, proximity to higher levels of care, and many other facets differ between outpatient facilities. Each of these patient selection criteria variables need to be considered in light of one's circumstances. Because of this, a one-size fits all criteria will never be appropriate. Initial patient selection criteria should be formalized based on thoughtful consideration of variables in light of the facility circumstances and should always be reviewed and modified as needed.

Failure of planned day of surgery discharge provides a list of care design aspects to optimize. $[\underline{1}, \underline{2}, \underline{5}, \underline{19}]$ These heed the importance of protocols that address postoperative pain, urinary retention, nausea/vomiting prevention and hypotension. They also highlight the importance of preoperative education, focusing on the need for a coach/ support staff at home, as well as the patient understanding of the logistics and time-course of the operative day's events.

Tables 1 and 2 can be used as a starting point for care teams to consider in creation of their patient selection criteria. As noted, the criteria variables can be best modified by teams with awareness of their unique circumstances. We recommend that a multispecialty group, including representatives from surgery, anesthesia, nursing, therapy and administration are present to provide input for discussion, and that thoughtful and logical selection criteria are formalized. These can then be implemented with careful tracking and refined within a multispecialty quality control process.

Table 3 presents optimization strategies for variables. These are valuable and the extent of which a care team desires to implement these aspects should be determined. For instance, a robust and high-volume program may have the ability to institute care design pathways for many of these issues, including obesity management, psychopa- 
Table 3: Variables to be considered for optimization during development of patient selection criteria.

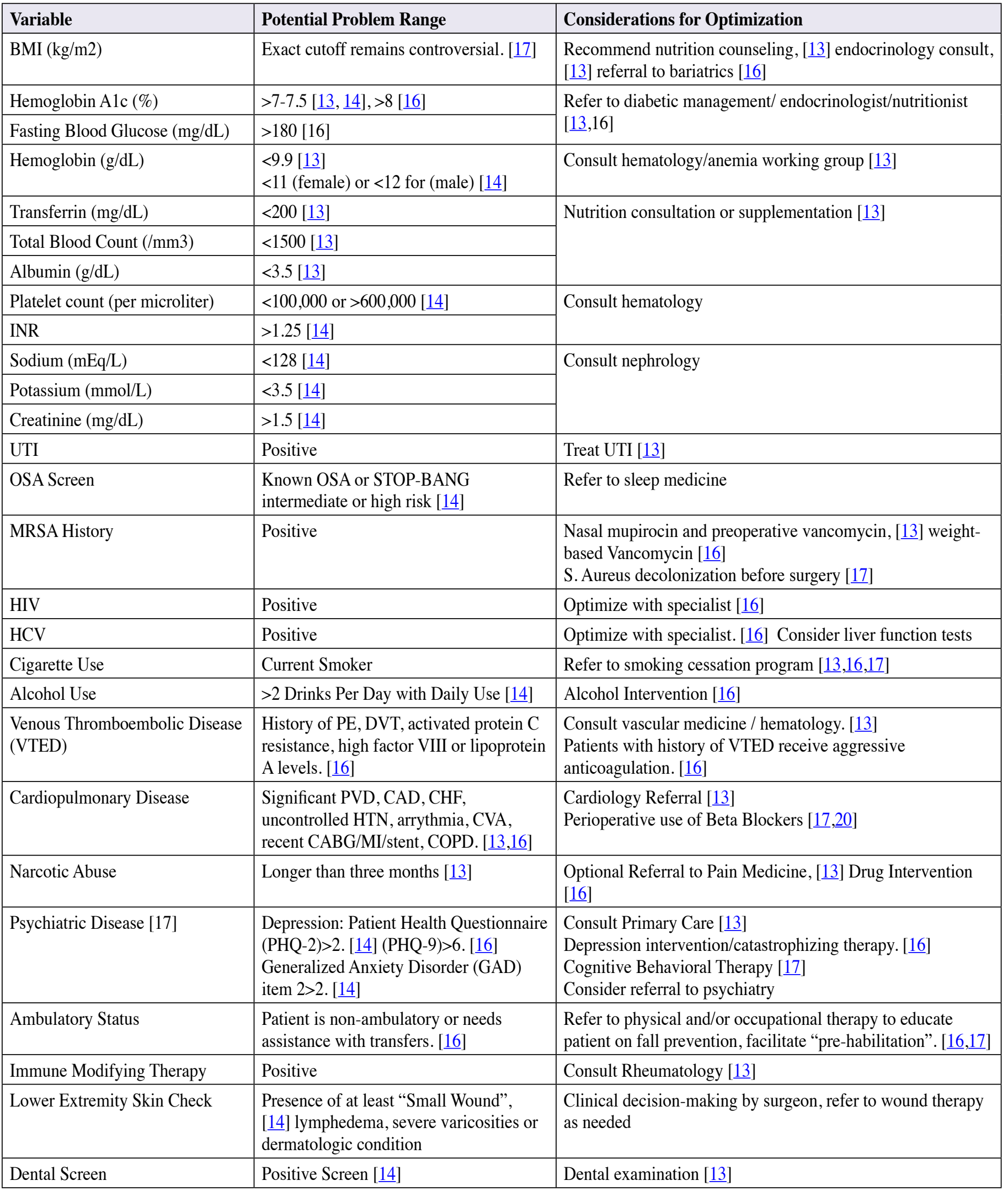

Abbreviations: Urinary Tract Infection (UTI), Obstructive Sleep Apnea (OSA) Pulmonary Embolism (PE), Deep Venous Thrombosis (DVT), Peripheral Vascular Disease (PVD), Coronary Artery Disease (CAD), Congestive Heart Failure (CHF), Hypertension (HTN), Cerebrovascular Accident (CVA), Coronary Artery Bypass Graft (CABG), Myocardial Infarction (MI), Chronic Obstructive Pulmonary Disease (COPD) 
thology and nutrition optimization. However, other programs may desire a focus on only cardiac optimization when needed, and an otherwise standard list of inclusion and exclusion criteria. More robust scoring systems can also be considered and will, necessarily and practically, be resource dependent.

\section{Conclusion}

Patient selection criteria are an important aspect of care design within a successful outpatient arthroplasty program. A review of outpatient TJA selection criteria, optimization strategies, and etiology of failed same day discharge is presented. We hope the synthesis of this data provides a starting point and valuable reference for care teams to aid creation of their specific, facility-appropriate, outpatient arthroplasty selection criteria.

\section{References}

1. Fraser JF, Danoff JR, Manrique J, Reynolds MJ, Hozack WJ. Identifying Reasons for Failed Same-Day Discharge Following Primary Total Hip Arthroplasty. J Arthroplasty. Dec 2018;33(12):3624-3628. doi:10.1016/j.arth.2018.08.003

2. Goyal N, Chen AF, Padgett SE, et al. Otto Aufranc Award: A Multicenter, Randomized Study of Outpatient versus Inpatient Total Hip Arthroplasty. Clin Orthop Relat Res. Feb 2017;475(2):364-372. doi:10.1007/s11999-016-4915-z

3. Weiser MC, Kim KY, Anoushiravani AA, Iorio R, Davidovitch RI. Outpatient Total Hip Arthroplasty Has Minimal Short-Term Complications With the Use of Institutional Protocols. J Arthroplasty. Nov 2018;33(11):3502-3507. doi:10.1016/j. arth.2018.07.015

4. Toy PC, Fournier MN, Throckmorton TW, Mihalko WM. Low Rates of Adverse Events Following Ambulatory Outpatient Total Hip Arthroplasty at a FreeStanding Surgery Center. J Arthroplasty. Jan 2018;33(1):46-50. doi:10.1016/j. arth.2017.08.026

5. Richards M, Alyousif H, Kim JK, Poitras S, Penning J, Beaule PE. An Evaluation of the Safety and Effectiveness of Total Hip Arthroplasty as an Outpatient Procedure: A Matched-Cohort Analysis. J Arthroplasty. Oct 2018;33(10):3206-3210. doi:10.1016/j.arth.2018.05.036

6. Klingenstein GG, Schoifet SD, Jain RK, Reid JJ, Porat MD, Otegbeye MK. Rapid Discharge to Home After Total Knee Arthroplasty Is Safe in Eligible Medicare Patients. J Arthroplasty. Nov 2017;32(11):3308-3313. doi:10.1016/j. arth.2017.06.034

7. Kim KY, Feng JE, Anoushiravani AA, Dranoff E, Davidovitch RI, Schwarzkopf R. Rapid Discharge in Total Hip Arthroplasty: Utility of the Outpatient Arthroplasty Risk Assessment Tool in Predicting Same-Day and Next-Day Discharge. J Arthroplasty. Aug 2018;33(8):2412-2416. doi:10.1016/j.arth.2018.03.025

8. Meneghini RM, Ziemba-Davis M, Ishmael MK, Kuzma AL, Caccavallo P. Safe Selection of Outpatient Joint Arthroplasty Patients With Medical Risk Stratification: the "Outpatient Arthroplasty Risk Assessment Score". J Arthroplasty. Aug 2017;32(8):2325-2331. doi:10.1016/j.arth.2017.03.004

9. Slover J. Same Day Total Joint Arthroplasty: How do I get Started? Lecture Presented At: Joint Arthroplasty Mountain Meeting; February 2020; Park City, UT

10. Kingery MT, Cuff GE, Hutzler LH, Popovic J, Davidovitch RI, Bosco JA. Total Joint Arthroplasty in Ambulatory Surgery Centers: Analysis of Disqualifying Conditions and the Frequency at Which They Occur. J Arthroplasty. Jan 2018;33(1):69. doi:10.1016/j.arth.2017.07.048

11. O'Neill O. Developing a Commercial Bundle. Twin Cities Orthopedics; 2016.

12. NYU Langone Medical Center's TJA BPCI Experience: Lessons in How to Maximize Value. Healthcare. Slideshare.net. November 16, 2016. Accessed February 8, 2021. https://www.slideshare.net/wellbeme/nyu-langone-medical-centers-tjabpci-experience-lessons-in-how-to-maximize-value
13. Featherall J, Brigati DP, Arney AN, et al. Effects of a Total Knee Arthroplasty Care Pathway on Cost, Quality, and Patient Experience: Toward Measuring the Triple Aim. J Arthroplasty. Nov 2019;34(11):2561-2568. doi:10.1016/j.arth.2019.06.011

14. Bernstein DN, Liu TC, Winegar AL, et al. Evaluation of a Preoperative Optimization Protocol for Primary Hip and Knee Arthroplasty Patients. J Arthroplasty. Dec 2018;33(12):3642-3648. doi:10.1016/j.arth.2018.08.018

15. Backstein D, Thiagarajah S, Halawi MJ, Mont MA. Outpatient Total Knee Arthroplasty-The New Reality and How Can It Be Achieved? J Arthroplasty. Dec 2018;33(12):3595-3598. doi:10.1016/j.arth.2018.09.042

16. Kim KY, Anoushiravani AA, Chen KK, et al. Perioperative Orthopedic Surgical Home: Optimizing Total Joint Arthroplasty Candidates and Preventing Readmission. J Arthroplasty. Jul 2019;34(7s):S91-s96. doi:10.1016/j.arth.2019.01.020

17. Yu S, Garvin KL, Healy WL, Pellegrini VD, Jr., Iorio R. Preventing Hospital Readmissions and Limiting the Complications Associated With Total Joint Arthroplasty. J Am Acad Orthop Surg. Nov 2015;23(11):e60-71. doi:10.5435/jaaosd-15-00044

18. Boraiah S, Joo L, Inneh IA, et al. Management of Modifiable Risk Factors Prior to Primary Hip and Knee Arthroplasty: A Readmission Risk Assessment Tool. J Bone Joint Surg Am. Dec 2 2015;97(23):1921-8. doi:10.2106/jbjs.n.01196

19. DeCook CA. Outpatient Joint Arthroplasty: Transitioning to the Ambulatory Surgery Center. J Arthroplasty. Jul 2019;34(7s):S48-s50. doi:10.1016/j. arth.2019.01.006

20. Urban MK, Markowitz SM, Gordon MA, Urquhart BL, Kligfield P. Postoperative prophylactic administration of beta-adrenergic blockers in patients at risk for myocardial ischemia. Anesth Analg. Jun 2000;90(6):1257-61. doi:10.1097/00000539200006000-00001

21. Learmonth ID, Young C, Rorabeck C. The operation of the century: total hip replacement. Lancet. Oct 27 2007;370(9597):1508-19. doi:10.1016/s01406736(07)60457-7

22. Bumpass DB, Nunley RM. Assessing the value of a total joint replacement. Curr Rev Musculoskelet Med. Dec 2012;5(4):274-82. doi:10.1007/s12178-012-9139-6

\section{SUBMISSION HISTORY}

Submitted: February 24, 2021

Reviewed: April 21, 2021

Accepted: September 29, 2021

Published: October 5, 2021

\section{AUTHOR AFFILIATIONS}

1 Dr. Joseph Gondusky, MD Jordan-Young Institute, 5716 Cleveland St \#200, Virginia Beach, VA

2 Richard Pahapill, MD; Christian Coulson, MD Eastern Virginia Medical School, 825 Fairfax Ave, Norfolk, VA (Direct inquires to Dr. Joseph Gondusky, josephgondusky@gmail.com)

AUTHOR DISCLOSURES

- The authors declare that there is no conflict of interest in connection with this submitted article.

\section{COPYRIGHT \& OPEN ACCESS}

(c) 2021 Gondusky, Pahapill, Coulson. All rights reserved. Authors retain copyright and grant the journal right of first publication with the work. Reconstructive Review is an open access publication and follows the Creative Commons Attribution-NonCommercial CC BY$\mathrm{NC}$. This license allows anyone to download works, build upon they credit the senior author, Reconstructive Review, and the Joint Implant Surgery \& Research Foundation (JISRF). An example credit would be: "Courtesy of (senior author's name), Reconstructive Review, JISRF, Chagrin Falls, Ohio". 